\title{
EL ARTE DE CURAR Y DE CUIDAR DESDE EL PUNTO DE VISTA TERMINOLÓGICO
}

Jesús Prieto Moreno. Profesor Asociado E.U.E. de Plasencia. Universidad de Extremadura Antonio Galindo Casero. Profesor Titular E.U.E. de Cáceres. Universidad de Extremadura

\author{
Jesús Prieto Moreno \\ C/. Consuegra, 20. 10600 Plasencia (Cáceres) \\ Telf: 927412678 - Fax: 927423422 \\ E-mail: jesusprieto@unex.es
}

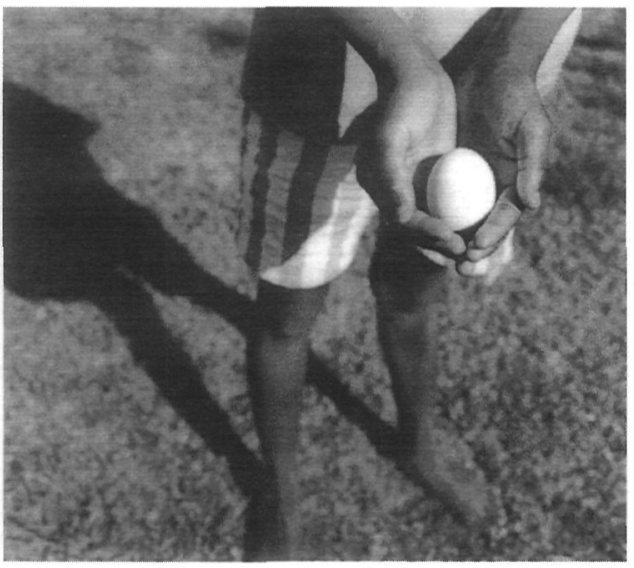

\section{RESUMEN}

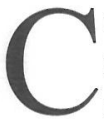

on el presente trabajo, hemos pretendido realizar un estudio, sobre la terminología más frecuente que utilizaba la Enfermería en la época que comprende los siglos XVI y XVII.

Cuando llevamos a cabo la clasificación y el significado de cada uno de los términos sanitarios, hay muchos de estos que nos permiten extraer conclusiones sobre las diversas artes de curar y cuidar que se practicaban, además, obtenemos de paso una buena visión de la enfermería y la medicina de la época.

Términos que nos hablan de técnicas y procedimientos, materiales, instrumentos diversos, preparación, indicación y administración de los distintos preparados medicinales, enfermedades que tra- taban, algunas de ellas desconocidas ya en nuestros días, todos los términos son un magnífico exponente de la Enfermería de los siglos XVI y XVII, sus limitaciones, su reconocimiento, sus funciones, etc.

Dentro de las conclusiones, a destacar la utilización de diferentes teorías, la influencia religiosa, las creencias populares, etc todas ellas se basaban en la influencia que tenían sobre las enfermedades.

\section{THE CRAFT OF HEALING AND CARING FROM THE PERSPECTIVE OF TERMINOLOGY}

$\mathrm{W}$ ith this work we intend to carry out a study about the most frequent terminology used by nurses during the XVIXVII century.

According to classification and meaning of each health-related word, many of them allow us to come to conclusions about the various crafts of healing and caring and subsequently to obtain a good perspective of nursing and medicine during that period.

All these terms and words about techniques, procedures, administration of the different medicines, diseases -some of then unknown in our days-, clarify the role of nurses in those centuries, their limitations and recognition.

Among the conclusions, the relevance of the different theories, religion and popular beliefs is remarked upon. 


\section{EL ARTE DE CURAR Y DE CUIDAR DESDE EL PUNTO DE VISTA TERMINO- LOGICO}

\section{INTRODUCCION Y OBJETIVOS}

\section{$\tau$}

os estudios sobre el cuidador, es decir, la persona que asiste al enfermo durante todo el proceso de su enfermedad, y que es un personaje tan antiguo como la enfermedad misma, han tropezado con dificultades cuando intentamos acercarnos a ellos.

Durante los siglos XVI y XVII, la Enfermería logra grandes avances, produciéndose transformaciones importantes en la misma, que la llevan a establecerse como un oficio definido y diferenciado de otras actividades profesionales con las que se encontraba relacionadas: barbero, cirujano menor, flebotomista, etc.

Desde mediados del siglo XVI hasta mediados del siglo XVII, sucedieron una serie de hechos que impulsaron de forma considerable la Enfermería: En primer lugar la Constitución y expansión de las Órdenes religiosas, en segundo lugar la Composición de manuales y textos de Enfermería escritos por y para enfermeros y en tercer lugar el desarrollo de las funciones de enfermería.

Aparece por tanto en ésta época, manuales orientados a la formación de los enfermeros, que intentan delimitar los conocimientos propios y en gran medida la definición de la profesión, además de liberar a las prácticas asistenciales del gran número de supersticiones y creencias que poblaban las artes curativas, quedando éstas reducidas a un mínimo número.

Las citadas obras, aparte de que representan un gran salto para el desarrollo de la Profesión, su lectura nos ha llevado a lograr una buena visión de la enfermería y la medicina de la época, dentro de ella nuestro objetivo ha sido:

- Centrarnos en el estudio, clasificación y significado de los términos más frecuentes que utilizaban en la época.

- Encontrados los términos, sacar conclusiones sobre las diversas artes de curar que poseían.

\section{MATERIAL Y METODOS}

Los resultados que presentamos se han obtenido del análisis de textos y manuales, algunos de ellos reproducciones actuales de los siglos XVI y XVII.

\section{RESULTADOS}

Hemos extractado dentro de lo posible, un número de términos significativo, sobre la nomenclatura que se utilizaba tanto de tratamientos, anatomía, cuidados, curaciones, etc. La mayoría de los términos, representan técnicas de Enfermería, en las cuales se utilizan expresiones que en nuestros días están fuera de uso o han dado lugar a formaciones nuevas.

\section{TÉRMINOLOGÍA}

ABSINTINA.- Es un principio amargo y tóxico del ajenjo, planta empleada principalmente como tónica y estimulante que puede administrarse en formas de polvo en infusión, cocimiento o de vino y con agua destilada.

Aceite de ALACRANES.- Se prepara echando alacranes vivos en aceite y esperando a que se ahoguen; después se añade agua, se calienta la mezcla hasta evaporar la humedad y se filtra con papel; es considerado bueno, untado en el vientre para promover la orina. Entre otros aceites, encontramos el de alcaparra, almendras, eneldo, laurel, lombrices, manzanilla, violado, rosado, Mathiolo, costo, común, etc.

Aceite de CHAULMOGRA.- Aceite vegetal que se obtiene de las semillas de la ginocardia odorata. Es utilizado en el tratamiento de la lepra.

Aceite de QUENOPODIO.- Aceite vegetal utilizado en afecciones parasitarias por vermes (ascaris, anquilostomas).

AGUA DE VEGETO.- Agua de color blanco lechoso, preparada a base de subacetato de plomo y que se usa en aplicaciones externas, por ser tóxi$\mathrm{ca}$, en forma de compresas húmedas, en contusiones y otros procesos por su poder astringente, que hace disminuir el derrame sanguíneo en los tejidos.

ALGOR.- Frialdad, escalofríos.

ANHÉLITO CORRUPTO.- Aliento o respiración fatigosa, penosa, alterada.

APOPLEXÍA (apoplejía).- Complejo sinto- 
mático que se caracteriza por la abolición del funcionalismo cerebral producido por diversas causas, especialmente por la embolia y hemorragia, de la que el término es sinónimo.

APOSTEMA.- Absceso. Acumulación localizada en una cavidad orgánica noviformada. Recibe diferentes nombres según su topografía.

AVERTINA.- Sustancia usada como anestésico y administrada en enemas por vía rectal.

AYUDA LENITIVA.- La que tiene como fin ablandar, suavizar.

AZOGUE.- Mercurio.

BÁLSAMO.- Exudación procedente de ciertos vegetales, cuya composición común puede representarse por resina, ácido benzóico o cinámico, y aceite volátil.

En farmacia se denomina también así muchos preparados en que entran sustancias aromáticas. Se emplean los naturales, tanto al interior como al exterior, y algunas veces en fumigaciones, y los artificiales generalmente al exterior como vulnerarios, fundentes y resolutivos.

BAZUQUEO.- Ruido hidroaéreo, se considera normal después de las comidas y de valor patológico cuando aparece alejado de las comidas o en ayunas.

BEBIDA CORDIAL.- Bebida obtenida de una mezcla de flores que en infusión se da a los enfermos como sudorífico.

BENTOSA (ventosa).- Vaso o campana, comúnmente de vidrio, que se aplica sobre una parte cualquiera de los tegumentos, después de haber enrarecido el aire en su interior quemando una cerilla o estopa, etc. La porción de tegumento substraído a la presión atmosférica se pone colorada y se entumece por el natural aflujo de humores. También se enrarece el aire por medio de una bomba aspirante adaptada al cuello de la ventosa. Las ventosas se han denominado secas cuando se aplican sobre una porción de la piel en que no se hayan hecho incisiones ni se hayan puesto sanguijuelas, es decir, que se aplican con el sólo objeto de producir allí una congestión artificial. Cuando se aplican donde se han puesto sanguijuelas o se han hecho escarificaciones o sajas y se favorece la salida de la sangre, se las llama sajadas o escarificadas.
BEXICATORIO (vejigatorio).- Dícese del emplasto de cantáridas u otra sustancia irritante, que levanta vejigas en la piel.

BEZOAR.- Piedra Bezahar. Cálculos especiales que se hallan en los estómagos de diferentes animales, principalmente de rumiantes. Antiguamente se atribuían a estos cálculos grandes virtudes curativas (contraveneno); hoy sólo tienen para la farmacia un interés histórico.

BORBORIGMO.- Se dice del ruido intestinal debido a la mezcla de gases y líquidos en el intestino y a su paso rápido por éste. Vulgar ruido de tripas.

BUBA.- Nombre vulgar de las pústulas. Mal venéreo o sifilítico. Tumores inflamatorios en ingle, axila y cuello, normalmente ligados a la sífilis. Las bubas pestilenciales son las derivadas de la peste.

CALA.- Supositorio rectal. Medicamentos oficinales o magistrales sólidos, de forma cónica alargada, a los que generalmente se da el grueso del dedo meñique, de la longitud de 3 a $5 \mathrm{~cm}$, y cuyo peso no debe exceder de 40 gramos. Fragmento de gasa que se coloca en una herida cavitaria para asegurar la libre salida de los productos morbosos.

CALCULO ALVINO.- Concreción en el intestino formada por el endurecimiento de porciones de contenido fecal.

CÁMARA.- Flujo de vientre. Diarrea.

CANTÁRIDINA.- Base de diversos medicamentos que se usan en forma de ungüento, emplasto, tintura y papel epispástico. Es en extremo vesicante, muy venenosa, dificulta la circulación sanguínea y actúa sobre el sistema nervioso excitándolo y produciendo delirio.

CATAPLASMA.- Son mezclas medicamentosas en forma de pasta blanda, destinada al uso externo, que se aplican sobre la piel directamente o envueltas en una gasa. Su objeto es unas veces sencillamente la aplicación del calor húmedo, ya sea para conseguir una acción calmante o emoliente, ya para macerar la piel o para favorecer la colección del pus, y otras veces aplicar también sobre la piel sustancias revulsivas, antisépticas, etc.

CLISTER.- Ayuda. Lavativa. Enema. Inyección destinada especialmente a los intestinos 


\section{6 - Cultura de los Cuidados}

gruesos. Pueden componerse con casi todas las preparaciones medicinales, y sirven también para introducir en los intestinos sustancias alimenticias. Obra el enema por su cantidad, calidad, temperatura, fuerza de propulsión, lo mismo que por su permanencia.

COCIMIENTO.- El líquido que resulta de hervir en agua, durante más o menos tiempo, ciertas sustancias medicinales (leños, raíces, hojas, cortezas, etc.) o de simples alimentos. También se denomina caldo medicinal. El cocimiento de cebada es la famosa tisana, empleada para todo tipo de enfermedades, pero sobre todo para las del pecho. Cuando el palo de guayaco llega a España, su principal forma de uso es precisamente el cocimiento, buscando promover la sudoración del enfermo.

CÓliCA O COLICA PASSIO.- Dolor cólico. Malestar abdominal acompañado de dolores continuos, retortijones y vómitos a causa de una obstrucción intestinal que alcanza su máximo grado en el cólico miserere.

COLILLA DEL ESPINAZO.- Punta de la columna vertebral.

CORRIMIENTO.- Fluxión de humores en alguna parte del cuerpo. .

COSTILLAS FIRMES.- Costillas verdaderas.

COSTILLAS ORNACINAS (formacinas).Costillas falsas.

COSTILLAS MENDOSAS.- (Mendosa: mentirosa o equivocada). Costillas falsas.

ÇERRO (CERRO).- Espinazo o lomo.

ÇOLLIPO (zollipo).- Hipo.

DEFENSIVO.- Paño doblado y mojado en algún licor, que se aplica a alguna parte enferma del cuerpo. Vendaje o emplasto destinado a proteger las partes que cubre.

DiACATOLiCón.- Purgante que se hacía principalmente con hojas de sen, raíz de ruibarbo y pulpa de tamarindo.

DIAFINICÓN.- Purgante elaborado con pulpa de dátiles, que fue muy usado antiguamente para combatir los llamados cólicos de plomo. Contenía escamonea, jengibre, pimienta negra, canela, ruda, semilla de dauco de Creta y de hinojos.
DOLOR DE HIJADA.- En principio todo dolor agudo sentido en el espacio comprendido entre la cadera y las últimas costillas. El cólico nefrítico debió ser el dolor de hijada más frecuente.

EMBROCACIÓN.- Acción de verter lentamente un líquido sobre una parte enferma. Diferénciase de la fricción en que no se emplea fuerza alguna para facilitar su absorción o penetración. Es un método terapéutico menos usado que la fricción, por ser menos activo. Aunque, en general se dirige a la superficie cutánea, puede asimismo hacerse en las mucosas (bucal, nasal, vaginal, etc.).

EMPLASTO.- Medicamento sólido, parecido por su composición a los ungüentos, pero que difiere de ellos por su mayor consistencia, por ser aglutinante y no licuarse con el calor del cuerpo. El emplasto tiene por base la combinación del protóxido de plomo con los ácidos oléico, margáfico y esteárico. Se extiende, generalmente, en capas delgadas sobre una tela de lienzo, tafetán o sobre baldés. Su forma guarda proporción con la conformación exterior de la región sobre la que se aplica. Entre los más conocidos se hallan el matrical para problemas de la madre; el confotativo de Vigo; el diaquilón, mayor y menor; el estomaticón; el de ranas, el resolutivo, etc.

EMUNTORIO.- Se aplica al órgano o conducto excretorio. Glándulas de los sobacos, ingles y de la parte posterior de las orejas.

EPITIMA.- Preparación tópica diferente del ungüento y del emplasto. Comprende tres clases: el epitema liquido, que cuando está caliente constituye los llamados fomentos; el epitema blando, como los polvos de creta, arcilla y talco; y el epitema seco o polvos simples o compuestos envueltos en una gasa o cubierta protectora.

ESQUINANÇIA (esquinancia).- Sofocación por apostema en la garganta, que dificulta el paso del aire y de alimentos y que suele cursar con fiebre y tos. Equivalía a las actuales anginas.

ESTANGURRIA ( estrangurria). Enfermedad de las vías urinarias, consistente en una micción dolorosa gota a gota con tenesmo o pujo de la vejiga.

ESTERNUTATORIO.- Que hace estornudar.

ETICA (héctica).- Fiebre diaria remitente, 
acompañada de escalofríos, sudor profuso, frecuencia y debilidad del pulso, enflaquecimiento y diarrea, asociada con la tuberculosis o supuración interna.

EXANGUE.- Desangrado, sin sangre o fuerzas.

FIEBRES.- Consideradas enfermedades en sí mismas. Se clasifican en efímeras, que suelen durar uno o dos días; humorales, por inflamación de los humores, y éticas, capaces de secar los miembros. A partir de aquí, sus tipos se multiplicaban en cotidianas, diurnas, nocturnas, tercianas, cuartanas, pestilenciales etc. La terciana es aquella fiebre intermitente cuyos accesos se repiten al tercer día, siendo cada cuatro en las cuartanas.

FLAGELACION.- Golpeteo repetido en los músculos para acelerar en ellos la circulación sanguinea

FLUXIÓN DE SANGRE.- Congestión o hiperemia activa.

FOMENTACIONES.- Fomentos. Aplicaciones de líquidos a diferentes partes del cuerpo, con el objeto de que permanezcan en ellas por más o menos tiempo. Los fomentos se hacen generalmente con infusiones, cocimientos o líquidos vinosos, acéticos, etéreos y alcohólicos.

FORMICACIÓN.- Hormigueo. Parestesia, sensación anormal como de insectos caminando sobre la piel.

FREGACIONES.- Friegas. Consisten en hacer fricciones sobre toda la superficie del cuerpo o de una parte con la mano sola o con bayetas o cepillos finos, que son las friegas secas, o bien empapando estos cuerpos con vapores aromáticos, que se llaman aromáticas, o con sustancias estimulantes de forma líquida, que son las friegas húmedas. Las friegas secas tienen por objeto aumentar la secreción y la enervación de la piel por faltar o haberse acumulado al interior, desarrollando, además, por este medio, el calor de la piel.

GALÁPAGO.- Venda hendida por los extremos, formando cuatro ramales.

GALLILLO.- Úvula o campanilla.

GARROTE.- Ligadura fuerte que se da en los brazos o muslos, oprimiendo su carne. Instrumento de que se han servido los cirujanos por mucho tiempo, antes del uso del torniquete, para ejercer sobre los vasos abiertos una compresión capaz de detener la hemorragia.

GARROTILLO.- Difteria, crup, muy común en los niños, que suele ocasionar la muerte por asfixia.

GOTA ARTÉTICA O GOTA FRÍA.- Gota que se padece en los artejos (nudillos de los dedos). Dolores de las articulaciones de todo el cuerpo.

GOTA CORAL.- Denominación que suele darse a la epilepsia.

HIJADA.- Cada una de las dos cavidades simétricamente colocadas entre las costillas falsas y los huesos de las caderas. Dolor que se padece en dicha parte.

\section{HIJAR.- Ijada.}

HISOPILLO.- Muñequilla que se empapa en agua u otro líquido, y con la cual se humedece la boca y la garganta de los enfermos.

HYSIPULA (erisipela).- Enfermedad aguda febril y eruptiva, caracterizada por síntomas generales y la erupción de una o varias placas rojas, dolorosas, con edema o infiltración de los tejidos subyacentes, limitadas por un reborde bien manifiesto a la vista y al tacto. HUESO PETENN.- Pubis, parte inferior del vientre.

HUMAÇO.- Humo de cualquier cosa encendida que se aplica a las narices por remedio.

INTESTINOS GRÁCILES.- Intestinos delgados.

JUNTURA.- Articulación.

LAMEDOR.- Composición que se hace en la botica de varios simples con azúcar

$\mathrm{y}$ es de menor consistencia que el electuario y de más que el jarabe.

LECHINO.- Clavo de hilas que se introduce en las úlceras y heridas para facilitar la supuración.

LETUARIOS (electuarios).- Son preparados de consistencia blanda y compuestos por lo general de polvos o extractos reunidos mediante un jarabe, azúcar, miel o conserva. A veces forman parte de ellos las pulpas, extractos, gomo-resinas, algunas sales, etc. Se les designa también con el 
nombre de confecciones, y cuando contienen alguna cantidad de opio, con el de opiatas. Son muy célebres y antiguos la triaca, el más potente antídoto conocido; el diascordio y el filonio romano, un opiáceo. Abundan en las boticas, siendo más comunes los denominados simple, diaprunis simple, diaprunis laxativo, rosado, indo, confección Hamech, diacatholicón, diafenicón y benedicta.

LINIMENTOS.- Consisten en líquidos untuosos, por lo general oleosos, que sirven para friccionar diversas partes de la piel con hilas, franelas, la mano, etc., con el objeto de mitigar ciertos padecimientos. La base de estos medicamentos la constituyen álcalis, sales, ácidos, jabones, bálsamos, etc. y su escipiente más común los aceites fijos y las grasas.

LLAMAMIENTO.- Acción de atraer humores de una parte del cuerpo a otra.

MODORRA.- Sueno pesado, sopor, letargo leve.

MORBO GÁLICO O MAL FRANCÉS.Sífilis. Enfermedad venérea.

MORCILLO (morcillo).- Parte carnosa o musculosa del brazo.

OPILACIÓN (opilación).- Obstrucción de cualquier vía u órgano hueco.

PAROXIMO (paroxismo).- Máxima intensidad de un acceso o ataque o de los síntomas de una enfermedad. Exacerbación súbita.

PASMO.- Lipotimia. Desmayo, deliquio, pérdida súbita del conocimiento.

PEBETE.- Pasta aromática que encendida exhala un humo muy fragante, y se usa para ahumar habitaciones.

PERLESÍA.- Parálisis. Debilidad muscular, senil o de otra clase, y acompañada de temblor.

PÍLDORAS.- Porción de electuario endurecida con polvos y de poco tamaño -cuando la porción era de mayor tamaño y más blanda se llamaba bolo.--, de uso frecuente. En cualquier botica podían hallarse las denominadas arábigas, de ruibarbo, aureas, de flumaria, de agárico y otras.

PIRINEO (perineo).- Región que forma el suelo de la pelvis y que comprende no sólo los diferentes planos músculoaponeuróticos, sino también los conductos urogenital y rectal que la atraviesan.
POLVOS.- Sirven para confeccionar preparaciones como las píldoras y para aplicación externa; los hay hemostáticos, aplicados a heridas sangrantes y amputaciones; afrodisíacos, como los polvos de cantárida, del español Juan de Vigo, compuestos de mercurio calcinado y agua fuerte, destinados a carnosidades y carne corrupta. Todo tipo de llagas corruptas, cánceres, enfermedades de la piel y parásitos eran tratados con polvos.

POMA.- Especie de bola elaborada con varios ingredientes, por lo común odoríferos. Perfumador.

PUXO (pujo).- Dolor abdominal acompañado de falsa necesidad de evacuar el vientre, con sensación de calor o escozor en la región anal. Contracción voluntaria o involuntaria de la prensa abdominal que acompaña la contracción uterina durante el período expulsivo del parto.

RABADILLA (diminutivo de rabada).Punta o extremidad del espinazo, formada por la última pieza del hueso sacro y por todas las del coxis.

REGUELDOS.- Eructo. Ventosidades humosas, por indigestión del estómago, expulsadas por la boca.

RESTAÑAR LA SANGRE.- Estancar, parar o detener la sangre.

SAHUMERIO.- Humo producido por una substancia aromática que se quema para perfumar o purifcar.

SANGUJA.- (Del latín sanguisuga; de sanguis, sangre, y sugere, chupar). Sanguijuela.

SÇIRRO (escirro).- Cáncer duro con predominio notable del tejido conjuntivo.

SECAS.- Apostema de la peste, bubón.

SIESSO (sieso).- Parte inferior del intestino recto en la cual se comprende el ano.

SINGULTO.- Hipo procedente de contracciones del estómago causadas por calor o frío o por mordicación producida por humores y alimentos inadecuados.

SPONDILES (espondiles).- Vértebras.

TABARDILLO.- Calentura pestilente con abundancia de sangre que se manifiesta por manchas en la piel; hoy se piensa que se trataba del tifus exantemático. 
TISANA.- Medicamento líquido que contiene en disolución una corta cantidad de principios medicinales, que sirve de bebida usual a los enfermos. Se administran frías o calientes, por cortadillos o tazas y a dosis variable.

TÍSSICA (tisis).- Enfermedad en que hay consunsión gradual y lenta, fiebre héctica y ulceración en algún órgano. Tuberculosis pulmonar.

TROÇISCOS (trociscos). Son medicamentos oficinales sólidos compuestos de una o más sustancias pulverizadas y unidas por un intermedio no azucarado como un mucílago, zumo vegetal, etc., y a los que se da la forma cónica, cúbica o piramidal. Similares a las tabletas y a las pastillas, se diferencian por el hecho de no tomar como base el azúcar, sino otro cualquier producto, como por ejemplo la miga de pan.

UNGÜENTOS.- Medicamentos oficinales compuestos, grasos y resinosos, de consistencia por lo común pastosa y que sólo se usan al exterior. Se diferencian de las pomadas en que éstas son más blandas y no contienen sustancias resinosas, y de los emplastos en que éstos son más consistentes y se aglutinan. Se emplean en fricciones o extendidos sobre lienzo, piel o hilas.

Ungüento BASILICÓN.- El madurativo y supurativo cuyo principio medicinal es la pez negra.

Ungüento DIALTEA.- Ungüento de malvavisco.

Ungüiento POPULEÓN.- Ungüento compuesto de manteca de cerdo, hojas de adormidera, belladona, yemas de chopo y otros ingredientes, el cual se emplea como calmante. En la farmacopea española (VII edición) es designado con el nombre de pomada de yemas de álamo compuesto.

XALEA (jalea).- Cualquiera de los medicamentos azucarados que tienen por base una materia animal o vegetal que al enfriarse toman consistencia gelatinosa.

XULEPE ( julepe). Porción compuesta de agua, goma, jarabe de goma y agua de azahar. Los antiguos daban este nombre a un medicamento líquido compuesto de tres partes de agua destilada aromática y 12 de azúcar. Se administra a cucharadas.

\section{CONCLUSIONES}

Dentro de la terminología estudiada, podemos constatar a través de los significados de los distintos términos las siguientes conclusiones:

- Utilización de plantas medicinales y animales para curar en determinadas ocasiones.

- El uso de la teoría de los colores: mediante la cual un color puede influir de forma positiva sobre enfermedades.

- El uso de la teoría de la gradación: se establecen cuatro grados, según sean más calientes o más fríos.

- La importancia del conocimiento de la orina para el diagnóstico y pronóstico de enfermedades.

- El fuerte arraigo de la astrología y la alquimia: las enfermedades siguen el curso de la luna o del sol.

- Vigencia de la teoría de los clásicos sobre los espiritus animales, como causa vital y origen de todo movimiento.

- Conjugación de la práctica con el empirismo, la influencia religiosa y las creencias populares con la aplicación de remedios caseros, alguno de ellos muy crueles.

- El uso de los tres pilares terapéuticos. Sangría, purga y dieta, los tres constituían los llamados remedios mayores de la medicina.

- La existencia de dudas, sobre la higiene en los preparados.

Las medidas terapéuticas empleadas, estaban en consonancia con el nivel de conocimientos que se poseía en ésta época sobre cuestiones sanitarias y al gran respeto que se tenía a muchas tradiciones y creencias populares.

\section{BIBLOGRAFIA}

- Alonso y Rodríguez, José.- "Compendio DE Terapéutica General". Carlos Bailly-Bailliere. Madrid, 1873.

- Diepgen, P.- " Historia de la Medicina". $3^{\circ}$ edición. Editorial Labor. Barcelona, 1.932.

- García Martínez, A.C; García Martínez, M.J; Valle Racero, J.I.- Presentación y análisis de la obra "Directorio de Enfermeros" de Simón López, siglo XVII. Editado por el Consejo General de Enfermería (Editorial Síntesis). Madrid, 1.997.

- García Martínez, A.C; García Martínez, M.J; Valle Racero, J.I.- Presentación y análisis de la obra "INSTRUCCIÓN DE ENFERMEROS" de Andrés Fernández de 1.625. Editado por el Consejo General 
de Enfermería (Editorial Síntesis). Madrid, 1.993.

- Granjel, Luis S.- "Historia dE LA Medicina ESPAÑOLA". SAYMA Ediciones y Publicaciones. Barcelona, 1.962.

- Laín Entralgo, P.- "Historia de la Medicina". Salvat Editores. Barcelona, 1.982.

- Pimulier, F.S.- "Diccionario Enciclopédico Ilustrado de Medicina, Cirugía Y Especialidades". $1^{a}$ edición. Ediciones Alonso.
Madrid, 1.962.

- Pimulier, F.S.- "ApÉndicE DEL Diccionario EnCiclopédico Ilustrado de Medicina, Cirugía y EsPeCIALIDADES". $1^{\text {a }}$ edición. Ediciones Alonso. Madrid, 1.962.

- Real Academia Española.- "Diccionario DE LA Lengua Española". Madrid, 1.992.

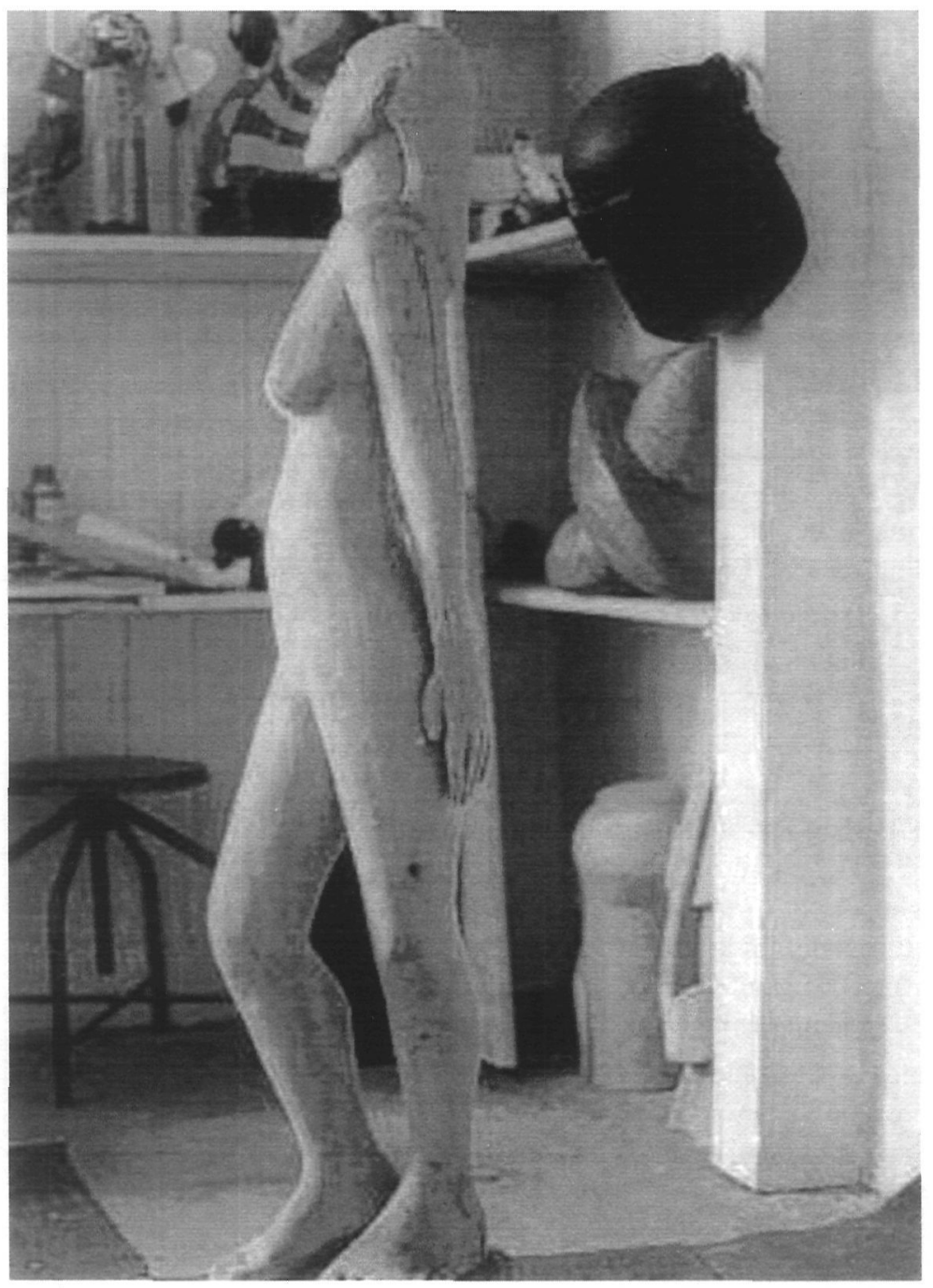

